tions of old estimators. Stat Med. 1999;18:695-706

8. Grunkemeier GL, Jin R, Eijkemans MJC, Takkenberg JJM. Actual and actuarial probabilities of competing risks: apples and lemons. Ann Thorac Surg. 2007; in press.

doi:10.1016/j.jtcvs.2006.03.069

\section{Atrial fibrillation surgery: Is it time to draw specific recommendations? To the Editor:}

We read with interest the article by Stulak and colleagues ${ }^{1}$ evidencing the limits of radiofrequency (RF) ablation compared with those of the maze technique. The reproduction by RF of the lesion pattern featured in the maze operation aims to obtain comparable results with shorter ischemic times and reduced incision-related bleeding risks. In fact, the results reported by the authors cast a shadow on this assumption; freedom from atrial fibrillation (AF) appears less satisfactory in patients treated with ablation compared with that seen in patients undergoing the classical maze operation, both at hospital discharge and at a median 8-month follow-up. ${ }^{1}$

The RF-based technique addresses the same atrial lesion pattern as that of the maze operation; subsequently, the different outcome of the treated patients is likely to be related to the efficacy of RF in terms of realization of transmural lesions. The purpose of RF ablation (ie, "to create full Cox maze lesions") ${ }^{1}$ is challenged.

The creation of transmural lesions is the aim of ablative surgery and should be considered a "must," ensuring procedural effectiveness. Unfortunately, intraoperative confirmations that this goal has been achieved are not available with the current methodologies, leaving a question mark on this issue, which is recalled by the results of Stulak and colleagues. ${ }^{1}$

Nevertheless, the widespread adoption of other-than-maze techniques (mostly based on the topical application of various energy sources to provoke conduction blocks) is a measure of how uncomfortable cardiac surgeons generally are with the maze operation. It also explains why the maze operation is adopted in few institutions of excellence and performed in selected patients instead of being a standardized and accepted procedure.

In fact, in daily practice cardiac surgeons are favoring less-invasive, more easily reproducible, and "softer" approaches, whose technology is in continuous evolution and whose efficacy is currently under evaluation.

The absence of guidelines or consensus statements, specifically those focusing on AF surgery, has been underlined by others. ${ }^{2,3}$ We believe that the extensive information gained from the clinical application of both the maze operation (since the early 1990s) and energy-based ablative surgery (since the late 1990s) would allow the cardiovascular community to draw recommendations indicating to surgeons when and how to proceed with AF surgery in patients undergoing cardiac operations. We are convinced that such recommendations would be fundamental to orientate both the cardiac surgeons in their daily practice and the related research in its evolution.

Francesca di Marco, PhD, MD Gino Gerosa, $M D$

Department of Cardiological, Thoracic, and Vascular Sciences Padua University Medical School Division of Cardiac Surgery Padova, Italy

\section{References}

1. Stulak JM, Dearani JA, Sundt TM 3rd, Daly RC, McGregor CG, Zehr KJ, et al. Superiority of cut-and-sew technique for the Cox maze procedure: comparison with radiofrequency ablation. J Thorac Cardiovasc Surg. 2007;133:1022-7.

2. Shemin RJ, Cox JL, Gillinov AM, Blackstone EH, Bridges CR, Workforce on Evidence-Based Surgery of the Society of Thoracic Surgeons. Guidelines for reporting data and outcomes for the surgical treatment of atrial fibrillation. Ann Thorac Surg. 2007;83:1225-30.

3. Khargi K. Why do we need an arrhythmia innovation center? Available at: http://www. ctsnet.org/sections/innovation/arrhythmia/articles/ article-1.html. Accessed May 2007. doi:10.1016/j.jtcvs.2007.05.070

\section{Reply to the Editor:}

We agree with Drs di Marco and Gerosa that it is time to synthesize the large amount of data on surgical treatment of atrial fibrillation (AF), conflicting as it might be, and formulate guidelines for clinicians. Fortunately, 2 recent publications address this need. The Society of Thoracic Surgeons Workforce on Evidence-based Surgery has recently published "Guidelines for reporting data and outcomes for the surgical treatment of atrial fibrillation."1
This group proposes standard descriptions of preoperative AF, of the surgical procedure, and of the lesion set performed. Furthermore, the guidelines propose uniform reporting of postoperative protocols, followup methodologies, and outcome rhythm.

A second publication by the Heart Rhythm Society Task Force on Catheter and Surgical Ablation of Atrial Fibrillation suggests the following indications for surgical treatment of AF: (1) symptomatic patients with AF undergoing other cardiac surgical procedures; (2) selected asymptomatic patients with AF undergoing cardiac surgery in whom the ablation can be performed with minimal risk; and (3) symptomatic patients with AF who prefer a surgical approach, have experienced 1 or more failed attempts at catheter ablation, or are not candidates for catheter ablation. ${ }^{2}$

Although surgical intervention for $\mathrm{AF}$ has been performed for 2 decades, prospective multicenter clinical trials are still needed to define the relative safely and efficacy of various surgical tools and techniques. In addition, surgeons should use consistent definitions of procedural success and follow-up methodology to compare the success of various surgical methods, as well as outcomes of surgical intervention, catheter ablation, and medical treatment.

John Stulak, MD

Hartzell V. Schaff, MD

Department of Cardiovascular Surgery Mayo Clinic College of Medicine

Rochester, Minn

\section{References}

1. Shemin RJ, Cox JL, Gillinov AM, Blackstone EH, Bridges CR. Workforce on EvidenceBased Surgery of the Society of Thoracic Surgeons. Guidelines for reporting data and outcomes for the surgical treatment of atrial fibrillation. Ann Thorac Surg. 2007;83: 1225-30.

2. European Heart Rhythm Association (EHRA), European Cardiac Arrhythmia Society (ECAS), American College of Cardiology (ACC), American Heart Association, Society of Thoracic Surgeons (STS), Calkins H, et al. HRS/EHRA/ECAS expert Consensus Statement on catheter and surgical ablation of atrial fibrillation: recommendations for personnel, policy, procedures and follow-up. A report of the Heart Rhythm Society (HRS) Task Force on catheter and surgical ablation of atrial fibrillation. Heart Rhythm. 2007;4: 816-61.

doi:10.1016/j.jtcvs.2007.07.058 